\title{
Large-scale Cosmic Ray Anisotropy with Tibet air shower array
}

${ }^{1}$ M. Amenomori, ${ }^{1}$ Y.-W. Bao, ${ }^{2}$ X. J. Bi, ${ }^{3}$ D. Chen, ${ }^{4}$ T. L. Chen, ${ }^{5}$ W. Y. Chen, ${ }^{3}$ Xu Chen, ${ }^{3,6}$ Y. Chen, ${ }^{2}$ Cirennima, ${ }^{5}$ S. W. Cui, ${ }^{7}$ Danzengluobu, ${ }^{5}$ L. K. Ding, ${ }^{3}$ J. H. Fang, ${ }^{3,}{ }^{6}$ K. Fang, ${ }^{3}$ C. F. Feng, ${ }^{8}$ Zhaoyang Feng, ${ }^{3}$ Z. Y. Feng, ${ }^{9}$ Qi Gao, ${ }^{5}$ Q. B. Gou, ${ }^{3}$ Y. Y. Guo, ${ }^{3}$ Y. Q. Guo, ${ }^{3}$ H. H. He, ${ }^{3}$ Z. T. He, ${ }^{7}$ K. Hibino, ${ }^{10}$ N. Hotta, ${ }^{11}$ Haibing Hu, ${ }^{5}$ H. B. Hu, ${ }^{3}$ J. Huang, ${ }^{3}$ H. Y. Jia, ${ }^{9}$ L.Jiang, ${ }^{3}$ H.-B. Jin, ${ }^{4}$ F. Kajino, ${ }^{12}$ K. Kasahara, ${ }^{13}$ Y. Katayose, ${ }^{14}$ C. Kato, ${ }^{15}$ S. Kato, ${ }^{16}$ K. Kawata, ${ }^{16}$ W. Kihara, ${ }^{15}$ Y. Ko, ${ }^{15}$ M. Kozai, ${ }^{17}$ Labaciren, ${ }^{5}$ G. M. Le, ${ }^{18}$ A. F. Li, ${ }^{19,8,3}$ H. J. Li, ${ }^{5}$ W. J. Li, ${ }^{3,9}{ }^{3}$ Y.-H. Lin, ${ }^{3,}{ }^{3}$ B. Liu, ${ }^{2}$ C. Liu, ${ }^{3}$ J. S. Liu, ${ }^{3}$ M. Y. Liu, ${ }^{5}$ W. Liu, ${ }^{3}$ Y.-Q. Lou, ${ }^{20}$ H.Lu, ${ }^{3}$ X. R. Meng, ${ }^{5}$ H. M itsui, ${ }^{14}$ K. Munakata, ${ }^{15}$ H. Nakada, ${ }^{14}$ Y. Nakamura, ${ }^{3}$ H. Nanjo, ${ }^{1}$ M. Nishizawa, ${ }^{21}$ M. Ohnishi, ${ }^{16}$ T. Ohura, ${ }^{14}$ S. Ozawa, ${ }^{22}$ X. L. Qian, ${ }^{23}$ X. B. Qu, ${ }^{24}$ T. Saito, ${ }^{25}$ M. Sakata, ${ }^{12}$ T. K. Sako, ${ }^{16}$ Y. Sengoku, ${ }^{14}$ J. Shao,,${ }^{3,} \mathrm{M}$. Shibata, ${ }^{14}$ A. Shiomi, ${ }^{26}$ H. Sugimoto, ${ }^{27}$ W. Takano, ${ }^{10} M$. Takita, ${ }^{16}$ Y. H. Tan, ${ }^{3}$ N. Tateyama, ${ }^{10}$ S. Torii, ${ }^{28}$ H. Tsuchiya, ${ }^{29}$ S. Udo, ${ }^{10}$ H. Wang, ${ }^{3}$ H. R. Wu, ${ }^{3}$ L. Xue, ${ }^{8}$ K. Yagisawa, ${ }^{14}$ Y. Yamamoto, ${ }^{12}$ Z. Yang, ${ }^{3}$ Y. Yokoe, ${ }^{16}$ A. F. Yuan, ${ }^{5}$ L. M. Zhai, ${ }^{4}$ H. M. Zhang, ${ }^{3}$ J. L. Zhang, ${ }^{3} X$. Zhang, ${ }^{2} X$. Y. Zhang, ${ }^{8}$ Y. Zhang, ${ }^{3}$ Yi Zhang ${ }^{1}{ }^{3}$ Ying Zhang, ${ }^{3}$ S. P. Zhao ${ }^{3}$ Zhaxisangzhu, ${ }^{5}$ and X. X. Zhou ${ }^{9}$ (The Tibet ASY Collaboration)

${ }^{1}$ Department of Physics, Hirosaki University, Hirosaki 036-8561, Japan

${ }^{2}$ School of Astronomy and Space Science, Nanjing University, Nanjing 210093, China

${ }^{3}$ Key Laboratory of Particle Astrophysics, Institute of High Energy Physics, Chinese Academy of Sciences, Beijing 100049, China

${ }^{4}$ National Astronomical Observatories, Chinese Academy of Sciences, Beijing 100012, China

${ }^{5}$ Physics Department of Science School, Tibet University, Lhasa 850000, China

${ }^{6}$ University of Chinese Academy of Sciences, Beijing 100049, China

${ }^{7}$ Department of Physics, Hebei Normal University, Shijiazhuang 050016, China

${ }^{8}$ Department of Physics, Shandong University, Jinan 250100, China

${ }^{9}$ Institute of Modern Physics, SouthWest Jiaotong University, Chengdu 610031, China

${ }^{10}$ Faculty of Engineering, Kanagawa University, Yokohama 221-8686, Japan

${ }^{11}$ Utsunomiya University, Utsunomiya 321-8505, Japan

${ }^{12}$ Department of Physics, Konan University, Kobe 658-8501, Japan

${ }^{13}$ Shibaura Institute of Technology, Saitama 337-8570, Japan

${ }^{14}$ Faculty of Engineering, Yokohama National University, Yokohama 240-8501,Japan

${ }^{15}$ Department of Physics, Shinshu University, Matsumoto 390-8621, Japan

${ }^{16}$ Institute for Cosmic Ray Research, University of Tokyo, Kashiwa 277-8582, Japan

${ }^{17}$ Institute of Space and Astronautical Science, Japan Aerospace Exploration

Agency (ISAS/JAXA),Sagamihara 252-5210, Japan

${ }^{18}$ National Center for Space Weather, China Meteorological Administration, Beijing 100081,

China

${ }^{19}$ School of Information Science and Engineering, Shandong Agriculture University, Taian 271018, China

${ }^{20}$ Physics Department, Astronomy Department and Tsinghua Center for Astrophysics, Tsinghua-

${ }^{1}$ Speaker

(c) Copyright owned by the author(s) under the terms of the Creative Commons 
National Astronomical Observatories of China joint Research Center for Astrophysics, Tsinghua University, Beijing 100084, China

${ }^{21}$ National Institute of Informatics, Tokyo 101-8430, Japan

${ }^{22}$ Advanced ICT Research Institute, National Institute of Information and Communication Technology, Koganei 184-8795, Japan

${ }^{23}$ Department of Mechanical and Electrical Engineering, Shandong Management University, Jinan 250357, China

${ }^{24}$ College of Science, China University of Petroleum, Qingdao, 266555, China

${ }^{25}$ Tokyo Metropolitan College of Industrial Technology, Tokyo 116-8523, Japan

${ }^{26}$ College of Industrial Technology, Nihon University, Narashino 275-8576, Japan

${ }^{27}$ Shonan Institute of Technology, Fujisawa 251-8511, Japan

${ }^{28}$ Research Institute for Science and Engineering, Waseda University, Tokyo 169-8555, Japan

${ }^{29}$ Japan Atomic Energy Agency, Tokai-mura 319-1195, Japan

E-mail: zhangyilihep.ac.cn

The large-scale sidereal anisotropy of cosmic rays is observed by Tibet air shower array in the northern hemisphere. Energy dependence of the cosmic-ray anisotropy from 300 $\mathrm{TeV}$ to $1 \mathrm{PeV}$ is analysed. We find that the anisotropy maps above $300 \mathrm{TeV}$ are distinct from that at the multi-TeV energy band. The spatial distribution of the GCR intensity of an excess and a deficit is observed in the $1 \mathrm{PeV}$ anisotropy map. All these results may further our understanding of the origin and propagation of GCRs. 


\section{Introduction}

The large-scale anisotropy of galactic cosmic rays (GCR) has been meticulously measured by underground and surface array experiments [1-9] in a wide energy range from sub $\mathrm{TeV}$ to a few PeV with the amplitude of the order of $10^{-4} \sim 10^{-3}$. Investigation of energy dependence of the $\mathrm{CR}$ anisotropy shows its amplitude increases with energy up to ten $\mathrm{TeV}$, and decrease at higher energies up to a few hundreds of TeV. Thanks to the two-dimensional high-precision analysis, a major change is found in the morphology of the anisotropy at the energy range of 100 300 TeV, indicating that the origin of the GCR anisotropy maybe different between multi-TeV and hundreds of $\mathrm{TeV}$.

In fact, the origin of GCR anisotropy is still unknown. The standard diffusive propagation model predicts one order of magnitude higher of the anisotropies compared with the measurements [10]; though this model can be effective in reproducing the spectrum and composition $[11,12]$. The predicted amplitude and the phase of the CR anisotropy are different from observations at multi-TeV energy. Some local conditions near the solar vicinity are introduced to reduce the predicted amplitude and to explain the phase, such as the local interstellar medium [13], local interstellar magnetic field [14], and the nearby source [15]. Noted that the predicted direction with enhanced CR intensity by the standard diffusion model is in the direction of the galactic center, due to the positional distribution of the GCR sources. This direction is contained in the relative excess region of the CR anisotropy observed above $300 \mathrm{TeV}$, which implies that the local environment may not dominate the anisotropy at around $\mathrm{PeV}$ energy. Therefore, the study of the CR anisotropy at PeV energy would be more important to obtain an improved understanding of the diffusion processes of GCR at energies close to the knee.

There have been only a few attempts to measure the CR anisotropy around knee energy, due to the rather low flux of CR at this energy. In the northern hemisphere, the EAS-TOP experiment presented the first detection of CR anisotropy at $\sim 200 \mathrm{TeV}$ but with limited statistics. Their updated results indicated some hints of increasing amplitude and change of phase at about a few hundreds of $\mathrm{TeV}$ [6]. The Tibet Air Shower (AS) array collaboration presented the first twodimensional anisotropy measurements from several $\mathrm{TeV}$ to several hundred $\mathrm{TeV}$ [1]. Their recent results revealed an excess region (with $7.2 \sigma$ pre-trial ) and a deficit region $(-5.8 \sigma$ pre-trial) in their $300 \mathrm{TeV}$ anisotropy map [2]. Hints of the existence of anisotropy at PeV were discussed as well in their analysis. In the southern hemisphere, the IceCube collaboration detected a distinct deficit with a post-trial significance of $-6.3 \sigma$ at $400 \mathrm{TeV}$ [16], which was then confirmed by IceTop. The Ice-Top experiment further uncovered the existence of anisotropy at energies up to 1 $\mathrm{PeV}[8]$.

This paper reports updated results on the CR anisotropy observed by Tibet air shower array at around PeV energy. It is based on 0.35 billion cosmic-ray events with energy above $300 \mathrm{TeV}$ recorded from 1997 May and 2017 May. The large size of the data set allows for a detailed study of the two-dimensional anisotropy.

\section{Experiment and analysis}

The Tibet AS array is located at Yangbajing in Tibet, China $\left(90.522^{\circ} \mathrm{E}, 30.102^{\circ} \mathrm{N}, 4300 \mathrm{~m}\right.$ above sea level, $606 \mathrm{~g} \cdot \mathrm{cm}^{-2}$ atmospheric depth). The surface array consists of plastic scintillation detectors with an area of $0.5 \mathrm{~m}^{2}$ each. The Tibet I array was constructed in 1990, with 65 plastic 
scintillation detectors placed on grids with $15 \mathrm{~m}$ spacing. It was later upgraded to 221 detectors, covering $36,900 \mathrm{~m}^{2}$, known as the Tibet II array. It began operation in 1995, with a trigger rate of $\sim 230 \mathrm{~Hz}$. The Tibet II was later upgraded to the current Tibet III, a denser array with $7.5 \mathrm{~m}$ grids, from 1999 to 2010 [17]. The trigger rate is $\sim 1700 \mathrm{~Hz}$ for the Tibet III array. From 2010, a waterCherenkov-type muon detector array was deployed underneath the surface scintillation array and started operation in 2014.

To obtain a long-term stable performance of the Tibet array, detectors noted as the Tibet II array are used in reconstruction in this paper throughout the observation period from 1995 October to 2017 May, with a few years (2011 2013) absent due to the upgrading. A standard shower reconstruction procedure is applied. Events are selected by imposing the following criteria consistent with previously work [2]:

(1) Four or more detectors should be fired; each fired detector should have more than 0.6 particle recorded;

(2) The reconstructed shower core should be located inside the array;

(3) Zenith angle $\theta<60^{\circ}$.

The energy of the primary CR is determined by two parameters: $\sum \rho_{\mathrm{FT}}$ ( sum of the number of particles per $\mathrm{m}^{2}$ counted by all the fast-timing detector) and the zenith angle $\theta$. $\sum \rho_{\mathrm{FT}}$ indicates the deposited energy in the array while $\theta$ represents the slant atmospheric depth where the CR travels through. Based on $\sum \rho_{\mathrm{FT}}$ and $\sec \theta$, a two-dimensional selection criterion has been developed for energy estimation by MC simulation. Event numbers in two energy bands are $3.6 \times 10^{8}(300 \mathrm{TeV})$, and $7.8 \times 10^{7}(1 \mathrm{PeV})$. Energy resolutions of each energy bands are estimated by $\mathrm{MC}$ simulation, details of which can refer to our previously work [2].

We analyze the data by employing the all-distance equi-zenith method $[1,18]$, which has been demonstrated to be sensitive for the observation of the large-scale anisotropy. Details of this method can be found in [18]. One-dimensional (1D) profile of the anisotropy is obtained by projecting the two-dimensional (2D) anisotropy map onto the right ascension (R.A.) axis, through averaging the relative intensities in all declinations. One-dimensional (1D) profile of the anisotropy can be fitted by the first-order harmonic function in the form of

$$
R(\alpha)=1+A_{1} \cos \left(\alpha-\phi_{1}\right),
$$

where $R(\alpha)$ denotes the relative intensity of CRs at R.A. $\alpha ; A_{1}$ is the amplitude of the firstorder harmonics; $\phi_{1}$ is the phase at which $R(\alpha)$ reaches its maximum.

\section{Results}

Figure 1(a) shows the significance map for the $300 \mathrm{TeV}$ energy, while Figure 1(b) shows the significance map for the $1 \mathrm{PeV}$ energy. The smoothing is then applied to the significance sky maps to improve the sensitivity for large features. The smoothing search applied in this analysis is from $25^{\circ}$ to $45^{\circ}$. In this work, $40^{\circ}$ is the optimized smooth radius for the $300 \mathrm{TeV}$ data set. The maximum significant features in the $300 \mathrm{TeV}$ map are found with an excess at $\left(\alpha=255^{\circ}, \delta=\right.$ $\left.13^{\circ}\right)$ with a significance value of $9.7 \sigma$, and a deficit at $\left(\alpha=71.7^{\circ}, \delta=3^{\circ}\right)$ with a significance value of $-6.7 \sigma$. The trial factor is estimated by assuming that all scans give statistically independent results. Because the optimization is performed over about $60 \times 180$ cells and 20 different smoothing radii, the total trial factor is expected to be about $2.16 \times 10^{5}$. The post-trial significance is $\sim 8.3 \sigma$ and $\sim-4.6 \sigma$ for the deficit regions, respectively. For the $1 \mathrm{PeV}$ map, same 
smoothing radius is employed. An excess centers at $\left(\alpha=264^{\circ}, \delta=-26^{\circ}\right)$ with a significance of $6.3 \sigma$ and a deficit centers at $\left(\alpha=91^{\circ}, \delta=-27^{\circ}\right)$ with a significance of $-5.6 \sigma$.
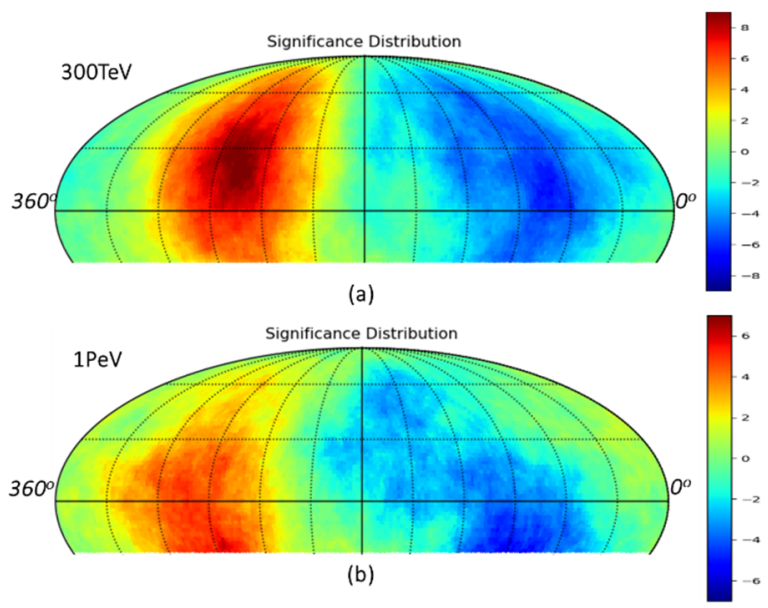

Figure 1. Panel (a), (b) show the pre-trial significance map for $300 \mathrm{TeV}$ and $1 \mathrm{PeV}$ energy bands plotted with $40^{\circ}$ smoothing, respectively.

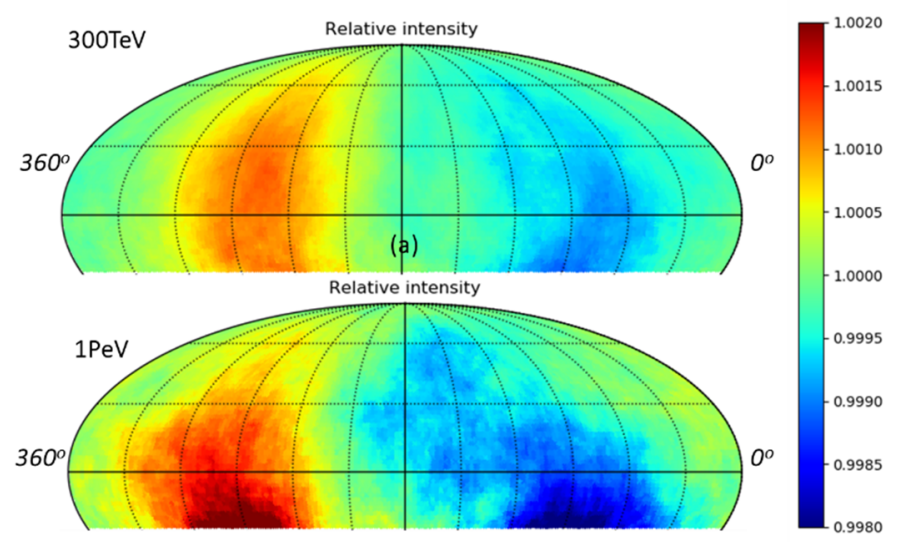

(b)

Figure 2. Panel (a), (b) show the relative intensity map for $300 \mathrm{TeV}$ and $1 \mathrm{PeV}$ energy bands plotted with $40^{\circ}$ smoothing, respectively.

Figure 2 (a) and (b) present the relative intensity map for the $300 \mathrm{TeV}$ and $1 \mathrm{PeV}$ energy bands, respectively. Because the acceptance of the detector decreases with the rise of the zenith angle, the relative intensity map is similar but not completely the same as the significance map. In figure 2, both the excess and deficit regions are consistent with that in the significance map for both energy bands.

Figure 3 (a) and (b) are the 1D projections of the relative intensity onto the R.A. before smoothing. The 1D projection can be fitted with the first-order harmonic function as shown in Equation 1. The blue curve shows the best-fitting result, with the fitting parameters indicated in the figure. The significance of non-zero amplitude is $6.3 \sigma$ for $300 \mathrm{TeV}$ data set, while $3.8 \sigma$ for 1 $\mathrm{PeV}$. The $\chi^{2}$ value is $34.9 / 16$ and $9.7 / 16$ for above data sets, respectively. It means that the firstorder harmonic function can describe the 1D projected profile well, indicating that a dipole structure is discovered in the CR anisotropy at energy above $300 \mathrm{TeV}$. 


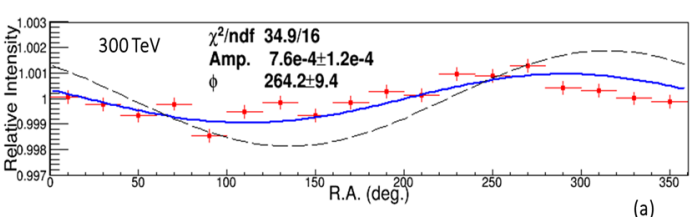

(a)

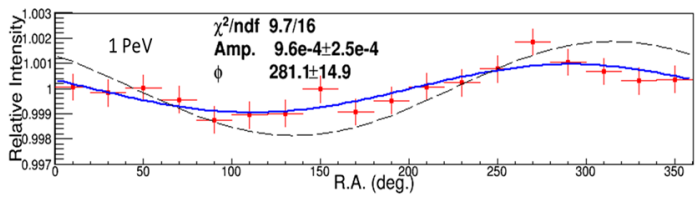

(b)

Figure 3. Panel (a) is the one-dimensional projection in the right ascension of the twodimensional map at energy of $300 \mathrm{TeV}$ in Figure 2(a). Panel (b) shows the one-dimensional projection in the right ascension of the $1 \mathrm{PeV}$ energy band of the two-dimensional map in Figure 2(b). The blue curve shows the first-order harmonic fitting to the data, while the black dashed line is the predicted Compton-Getting effect due to the orbital motion of the solar system around the galactic center.

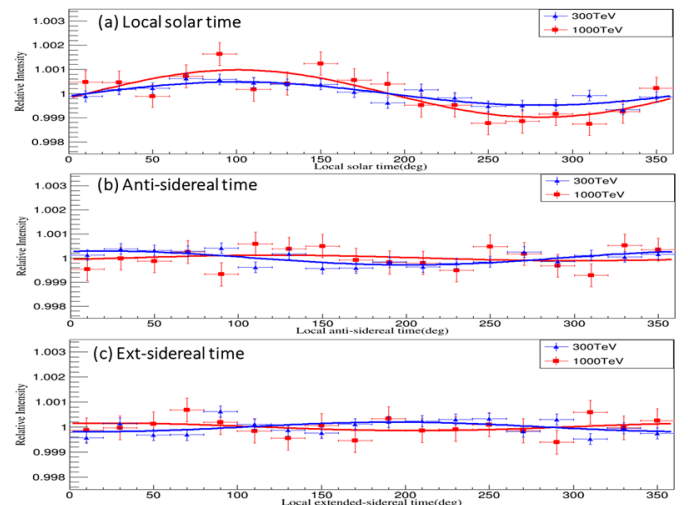

Figure 4. Panel (a) shows the one-dimensional projections of the two-dimensional anisotropy maps in local solar time. The blue dots indicate the one-dimensional projection in the energy band of $300 \mathrm{TeV}$, while the red dots are the onedimensional projection at $1 \mathrm{PeV}$ energy. The lines are the best-fitting result by the first-order harmonic function. Panel (b), (c) present the onedimensional projection results in anti-sidereal time and ext-sidereal time, respectively.

Table 1. Fitted results by the first-order harmonic function in local sidereal time and local solar time.

\begin{tabular}{ccccccc}
\hline $\begin{array}{c}\text { Energy } \\
\mathrm{TeV}\end{array}$ & $\begin{array}{c}A_{\text {sid }} \\
10^{-4}\end{array}$ & $\begin{array}{c}\phi_{\text {sid }} \\
{\left[{ }^{\circ}\right]}\end{array}$ & $\chi_{\text {sid }}^{2} / n d f$ & $\begin{array}{c}A_{\text {sol }} \\
10^{-4}\end{array}$ & $\begin{array}{c}\phi_{\text {sol }} \\
{\left[{ }^{\circ}\right]}\end{array}$ & $\chi_{\text {sol }}^{2} / n d f$ \\
\hline 300 & $7.6 \pm 1.2$ & $264.2 \pm 9.4$ & $34.9 / 16$ & $3.7 \pm 1.2$ & $98.1 \pm 20.2$ & $10.4 / 16$ \\
1000 & $9.6 \pm 2.5$ & $281.1 \pm 14.9$ & $9.7 / 16$ & $8.8 \pm 2.5$ & $97.7 \pm 16.1$ & $10.3 / 16$ \\
\hline
\end{tabular}

Table 2. Fitted results by the first-order harmonic function in ext-sidereal time and anti-sidereal time.

\begin{tabular}{ccccccc}
\hline \hline $\begin{array}{c}\text { Energy } \\
\mathrm{TeV}\end{array}$ & $\begin{array}{c}A_{\text {ext }} \\
10^{-4}\end{array}$ & $\begin{array}{c}\phi_{\text {ext }} \\
{\left[{ }^{\circ}\right]}\end{array}$ & $\chi_{\text {ext }}^{2} / n d f$ & $\begin{array}{c}A_{\text {anti }} \\
10^{-4}\end{array}$ & $\begin{array}{c}\phi_{\text {anti }} \\
{\left[{ }^{\circ}\right]}\end{array}$ & $\chi_{\text {anti }}^{2} / n d f$ \\
\hline 300 & $2.2 \pm 1.2$ & $202.5 \pm 31.8$ & $22.6 / 16$ & $2.6 \pm 1.2$ & $19.6 \pm 27.7$ & $13.2 / 16$ \\
1000 & $1.1 \pm 2.5$ & $338.8 \pm 135.8$ & $7.6 / 16$ & $1.1 \pm 2.5$ & $142.4 \pm 124.9$ & $11.8 / 16$ \\
\hline
\end{tabular}

In order to assess the systematic uncertainties of the sidereal anisotropy caused by the seasonal variation of the detectors, identical analyses are performed in local solar time, antisidereal time and extended-sidereal time for both energy bands. Figure 4 shows daily variations in three frames of time and the best-fit parameters are also shown in Table 1 and 2 . The amplitude and phase in the solar time are consistent with the expectation from the CG effect due to the terrestrial orbital motion around the Sun, where $A_{\text {sol, CG }}$ is $0.047 \%$ and $\varphi_{\text {sol, CG }}$ is at 6.0 hours. In both data sets, no significant anisotropy is observed in anti-sidereal time and ext-sidereal time, indicating systematic uncertainties is well controlled.

\section{Conclusion}


In this paper, we present the large-scale CR sidereal anisotropy at energy of about $300 \mathrm{TeV}$ and $1 \mathrm{PeV}$, based on $3.5 \times 10^{8} \mathrm{CR}$ events recorded by Tibet AS array from 1995 October to 2017 May. Energy evolution of the large-scale sidereal anisotropy has been obtained from $300 \mathrm{TeV}$ to $1 \mathrm{PeV}$. No major change is found in the morphology of the anisotropy at the energy range from $300 \mathrm{TeV}$ to $1 \mathrm{PeV}$. The sidereal anisotropy observed at $1 \mathrm{PeV}$ reveals a significant relative an excess centered at $\left(\alpha=264^{\circ}, \delta=-26^{\circ}\right)$ with a significance of $6.3 \sigma$ and a deficit centered at $\left(\alpha=91^{\circ}, \delta=-27^{\circ}\right)$ with a significance of $-5.6 \sigma$. In addition, positions of these hot spots are different from that appeared in the $300 \mathrm{TeV}$ map.

The relative intensity as a function of right ascension is fitted with the first-order harmonic function. The amplitudes and phases at $300 \mathrm{TeV}$ and $1 \mathrm{PeV}$ are summarized in Table 1 . The statistic is still not enough to recognize the difference between two energy bands on values of the phase and amplitude. The solar anisotropy expected from the Earth's revolution around the Sun is analyzed, the amplitude and phase of which agree with the expectation in both energy bands. Moreover, the anisotropy in anti-sidereal and extended-sidereal time are also checked, where no significant signal is observed. Observations have ensured the reliability of the sidereal anisotropy measurement for both $300 \mathrm{TeV}$ and $1 \mathrm{PeV}$ data sets.

\section{Discussion}

The observed sidereal anisotropy around $1 \mathrm{PeV}$ shows substantial differences with respect to that observed below $100 \mathrm{TeV}$, however, the origin of the PeV CR anisotropy is still unknown. If there were a relative motion of the observer with respect to the cosmic-ray plasma, then this would produce the Compton-Getting (CG) effect [19]. This effect expected from the orbital motion of the solar system around the galactic center is not observed. [1, 4, 16]. The black dash lines in Figure 3 show the expected 1D projection by this effect. On the other hand, the reference frame of the GCR is unknown; the CG effect could be one possible contribution. Moreover, the amplitude of the anisotropy caused by CG effect would be energy independent but sensitivity to the index of the CR energy spectrum.

As the significant excess region at energy above $300 \mathrm{TeV}$ is from the direction of the Galactic center, it might be a natural propagation consequence of the GCRs. Because the GCR sources are mainly located at the disk, the standard CR diffusion model predicts a dipole anisotropy in the Galactic center direction. In this case, the dependence of anisotropy amplitude over primary energy would be proportional to the diffusion coefficient $\mathrm{D}$, where $\mathrm{D}$ is assumed to increase with magnetic rigidity $\left(D \propto R^{\delta}\right)$.

The study of the rigidity evolution of the anisotropy at $\sim 1 \mathrm{PeV}$ can therefore provide a significant test on the diffusion models and an ability for the discrimination between above two possible explanations of the CR anisotropy. This challenges $\mathrm{CR}$ experiments to upgrade their detectors to achieve the ability of mass discrimination. Additionally, the CR anisotropy above $\mathrm{PeV}$ may be possibly associated with the knee of GCRs, measurement of which would further our understanding of the origin and propagation of GCRs.

\section{Acknowledments}

This work is partly supported by the National Key R\&D Program of China No. 2018YFA0404202, by a Grant-in Aid for Scientific Research on Priority Areas from the Ministry 
of Education, Culture, Sports, Science and Technology, by Grants-in-Aid for Science Research from the Japan Society for the Promotion of Science in Japan, and by the Grants from the National Natural Science Foundation of China and the Chinese Academy of Sciences. Yi Zhang is supported by the Natural Sciences Foundation of China No. 11775233. The collaborative experiment of the Tibet Air Shower Arrays has been performed under the auspices of the Ministry of Science and Technology of China and the Ministry of Foreign Affairs of Japan.

\section{References}

[1] M. Amenomori, et al., Anisotropy and Corotation of Galactic Cosmic Rays, Science 314, (2006) 439.

[2] M. Amenomori, et al., Northern Sky Galactic Cosmic Ray Anisotropy between 10 and 1000 TeV with the Tibet Air Shower Array, ApJ 836, (2017) 153.

[3] G. Guillian, et al., Observation of the anisotropy of $10 \mathrm{TeV}$ primary cosmic ray nuclei flux with the Super-Kamiokande-I detector, Phys. Rev. D 75, (2007) 062003.

[4] A. A. Abdo, et al., THE LARGE-SCALE COSMIC-RAY ANISOTROPY AS OBSERVED WITH MILAGRO, ApJ 698, (2009) 2121.

[5] B. Bartoli, et al., ARGO-YBJ OBSERVATION OF THE LARGE-SCALE COSMIC RAY ANISOTROPY DURING THE SOLAR MINIMUM BETWEEN CYCLES 23 AND 24, ApJ 809, (2015) 90.

[6] M. Aglietta, et al., EVOLUTION OF THE COSMIC-RAY ANISOTROPY ABOVE $10^{\wedge} 14 \mathrm{eV}$, ApJ 692, (2009) L130.

[7] M. G. Aartsen, et al., ANISOTROPY IN COSMIC-RAY ARRIVAL DIRECTIONS IN THE SOUTHERN HEMISPHERE BASED ON SIX YEARS OF DATA FROM THE ICECUBE DETECTOR, ApJ 826, (2016) 220.

[8] M. G. Aartsen, et al., OBSERVATION OF COSMIC-RAY ANISOTROPY WITH THE ICETOP AIR SHOWER ARRAY, ApJ 765, (2013) 55.

[9] A. U. Abeysekara, et al., All-sky Measurement of the Anisotropy of Cosmic Rays at $10 \mathrm{TeV}$ and Mapping of the Local Interstellar Magnetic Field, ApJ 871, (2019) 96.

[10] C. Evoli, et al., Common Solution to the Cosmic Ray Anisotropy and Gradient Problems, Phys. Rev. Lett. 108, (2012) 211102.

[11] A. W. Strong, I. V. Moskalenko and V. S. Ptuskin, Cosmic-ray propagation and interactions in the Galaxy, Annu. Rev. Nucl. Part. Sci. 57, (2007) 285.

[12] P. Blasi and E. Amato, Diffusive propagation of cosmic rays from supernova remnants in the Galaxy. I: spectrum and chemical composition, J. Cosmol. Astropart. Phys. 2012, (2012) 010.

[13] S. Redfield and J. L. Linsky, The Three-dimensional Structure of the Warm Local Interstellar Medium. II. The Colorado Model of the Local Interstellar Cloud, The Astrophysical Journal 534, (2000) 825.

[14] P. C. Frisch, et al., COMPARISONS OF THE INTERSTELLAR MAGNETIC FIELD DIRECTIONS OBTAINED FROM THE IBEX RIBBON AND INTERSTELLAR POLARIZATIONS, ApJ 724, (2010) 1473.

[15] W. Liu, Y.-Q. Guo and Q. Yuan, Indication of nearby source signatures of cosmic rays from energy spectra and anisotropies, 15.

[16] R. Abbasi, et al., OBSERVATION OF ANISOTROPY IN THE GALACTIC COSMIC-RAY ARRIVAL DIRECTIONS AT 400 TeV WITH ICECUBE, ApJ 746, (2012) 33.

[17] M. Amenomori, et al., Multi - TeV Gamma - Ray Flares from Markarian 421 in 2000 and 2001 Observed with the Tibet Air Shower Array, ApJ 598, (2003) 242.

[18] M. Amenomori, et al., A Northern Sky Survey for Steady Tera-Electron Volt Gamma - Ray Point Sources Using the Tibet Air Shower Array, ApJ 633, (2005) 1005.

[19] A. H. Compton and I. A. Getting, An Apparent Effect of Galactic Rotation on the Intensity of Cosmic Rays, Phys. Rev. 47, (1935) 817. 